\title{
A Survey on Traffic Control Mechanism for Emergency Vehicles
}

\author{
Diksha A. Chaudhari, Lakxmi S. Gadhari, Gaurav H. Damale, Abhijit R. Dutonde \\ Prof. Himanshu Joshi (Assistant professor) \\ Department Of Computer Engineering \\ Jspm's Imperial College of Engineering and Research, SPPU, Pune, India 412207
}

\begin{abstract}
Vehicles count has drastically increased in the recent decades. This causes heavy traffic on roads leading to non clear roads for emergency vehicles, which in turn causes deaths of people who could not reach hospitals in time. So there is a need of the system which can make use of GPS coordinates of the emergency vehicles and hospitals to which the vehicle is moving for making the roads empty for time being. There are various systems previously proposed for this feature. In this paper, we have done the survey of previously proposed systems and tried to overcome the flaws of those systems to propose the advanced and cost effective system in comparison to previous systems.
\end{abstract}

Key word: Intelligent Traffic Management, Traffic Congestion, Emergency Vehicle.

\section{INTRODUCTION}

The Internet of Things (IoT) is a network of objects that can communicate with each other. The IoT in traffic management plays an important role here by collecting data from various sources such as traffic cameras, vehicles ${ }^{\text {ee }} \mathrm{GPS}$, commuters $^{\text {ee }}$ mobile phones, sensors on the roads, passing vehicles and so on. Traffic IoT provides traffic information collection and incorporation, supportive processing and analysis in all categories of traffic information on roads in a large area automatically and intelligently ${ }^{[1]}$. Modern traffic management is evolving into an intelligent transport system based on IoT. IoT functions in traffic management such as Consolidate traffic data coming from different sources, Analyse traffic information to provide near -real-timeinsights, Monitor traffic operations and incidents, Support the storage and presentation of geographic information system (GIS).Essential characteristics of IoT such as Dynamic \& Self- Adapting, Self-Configuring, Interoperable Communication Protocols, Unique Identity, Integrated into Information Network. Traffic congestion is a severe problem in many modern cities around the world. Traffic congestion has been causing various critical problems and Encounters in the major and most populated cities. To travel to different places within the city is becoming more difficult for the travelers in traffic.

Owing tothese congestion problems, people lose time, miss Opportunities, and get frustrated IoT application in traffic management provides dynamic interaction between the components of a transport system, allows inter and intra vehicular communication, smart traffic control, smart parking, electronic toll collection system, logistics and fleet management, vehicle control and safety, road assistance.

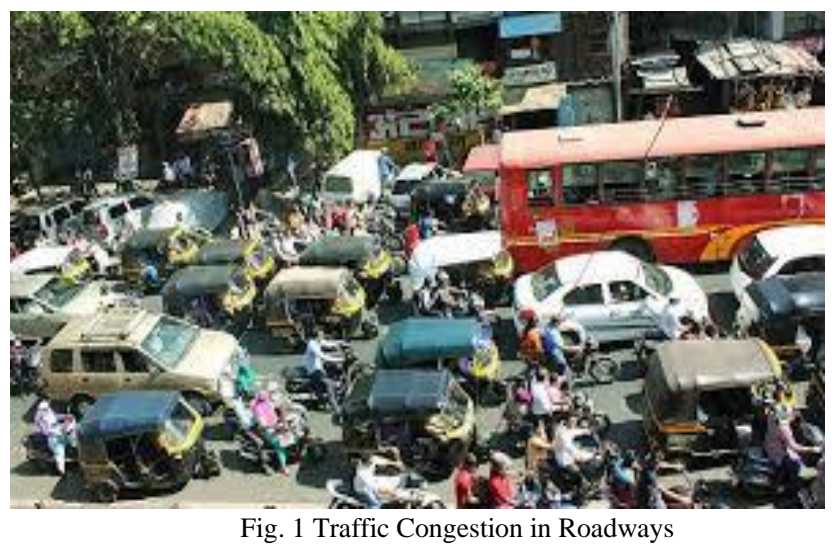

\section{LITERATURE SURVEY}

In paper[1] presents an Internet-of-Things-based objectives for emergency vehicle priority and self-organized traffic control (EVP-STC) management at intersections. The increase in number of motor vehicles, traffic jams in urban areas are creating a major problems. Traffic jams, especially those at junction, not only increase waiting time for drivers but also increase consumption of fuel and air pollution[1]. In paper[2], author proposed a new platform and protocol called EVP-STC that contains three main systems. The first system, called the intersection controller, is fixed at traffic signals and this gathers information about emergency vehicle position and vehicle density data at each road segment proceeding towards junction. The intersection controller then sets the timings of traffic signals based on detected real-time traffic. The second system is fixed at each road segment and this contains force resistive sensors to locate vehicles. It sends the gathered information to the intersection controller via ZigBee. The third system is fixed in emergency vehicles and this provides GPS coordinates to the intersection controller to avoid delay for emergency vehicles at junctions. Simulation results show the effectiveness of the proposed platform, which reduces total delays, lane opening times, and delay for emergency vehicles.

In paper[3] system takes input from traffic density as a) cameras b) and sensors, then control traffic signals. An additional algorithm based on Artificial Intelligence is used to predict the traffic density for future to reduce the traffic congestion. Apart from this, RFIDs are also used to prioritize the emergency vehicles such as ambulances and 
fire brigade vehicles during a traffic jam. Smoke sensors are also part of this system to detect the situation in case of fire on the road. To demonstrate the effectiveness of the proposed traffic management system, a prototype is developed which not only optimizes the traffic flow but also connects nearby rescue departments with a centralized server. Traffic seems to be an adaptation problem rather than an optimization problem The paper aims at examining methods to build an intelligent system that can combine and support some of the existing technologies of traffic control and therefore reduce the average waiting time of vehicles on a junction. The proposed algorithms are adjustable to flow of traffic at any junction point of roads. Simulations of the real-life traffic scenarios are conducted in a simulated platform called Green Light District Simulator (GLD) to generate graph average waiting time versus cycles. The results generated show that the proposed method is effective for the traffic control in a real road intersection.

In paper[4] author proposed the RFID tags for the traffic density measurement which leads to the usage of RFID readers to be mounted near every signal which is very cost increasing solution. So this system leads to lot of maintenance cost considering the realtime use of readers $24 \times 7 \&$ also the system requires additional hardware processors to be mounted at every signal for processing the RFID readers working.

In paper[5], the density of vehicles is checked by making use of IR sensors which are connected to the PIC controller. Which means that there will be need of multiple IR sensors at every signal connected roads, which is not a feasible solution because IR sensors have a very limited scope and very limited lifespan.

\section{CURRENT SYSTEM}

The current System focuses majorly on hardware to be used for GPS \& fetching \& the controller nodeMCU is then required to get connected to the cloud, so this causes the hardware cost to increase \& we cannot fit the same devices in all the existing vehicles. So there has to be a system which is cost efficient \& can also be used in existing vehicles which does not require any mechanical changes to be done in the vehicle.

\section{PROPOSED SYSTEM}

The proposed system will make use of the GPS integrated in the smartphones of the driving candidates which will help the traffic control systems to determine the density of the people on roads and accordingly can check the path of the emergency vehicle which they will receive from every emergency vehicle to reach the desired hospital and thereby make the route clear by making the timers of the corresponding signals green for $10 \mathrm{mins}$ prior to the emergency vehicle reaches the signal. The proposed system architecture is as follows:

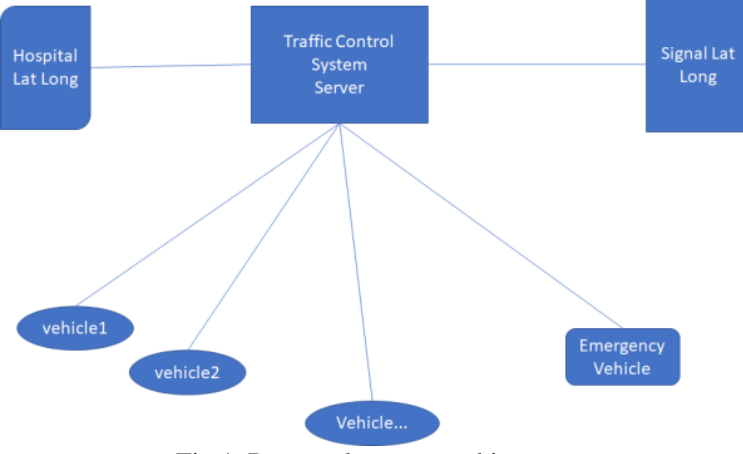

Fig 1. Proposed system architecture

\section{CONCLUSION}

From the survey done so far for the previous systems, It can be analysed that most of the systems make use of additional hardware and are not scalable to real time implementation. So we have proposed the advanced system which makes use of the integrated GPS module from smartphones to get the density near the signals having the specific radius of the latitude and longitude for every signal.

\section{REFERENCES}

[1] Ajmal Khan1, Farman Ullah1, Zeeshan Kaleem , Shams Ur Rahman, Hafeez Anwar1, And You-Ze Cho,"EVP-STC: Emergency Vehicle Priority and Self-Organising Traffic Control at Intersections Using Internet-of-Things Platform", Digital Object Identifier 10.1109/ACCESS.2018.2879644

[2] Sabeen Javaid, Ali Sufian, Saima Pervaiz, Mehak Tanveer, "Smart Traffic Management System Using Internet of Things", International Conference on Advanced Communications Technology(ICACT)

[3] Juhi R Srivastava, Dr. T. S. B. Sudarshan "Intelligent Traffic Management with Wireless Sensor Networks", 978-1-4799-07922/13/\$31.00 @2013 IEEE

[4] Tejas Naik, Roopalakshmi R, Divya Ravi N, Pawdhan Jain, Sowmya B H and Manichandra, "RFID-Based Smart Traffic Control Framework for Emergency Vehicles", Proceedings of the 2nd International Conference on Inventive Communication and Computational Technologies (ICICCT 2018)

[5] Bilal Ghazal, Khaled EIKhatib, Khaled Chahine, Mohamad Kherfan, "Smart Traffic Light Control System", ISBN: 978-14673-6942-8/16/\$31.00 @2016 IEEE

[6] A.S.Dhatrak, Dr.S.T.Gandhe, "Automatic Traffic Signals in Smart Cities for Speedy Clearance of Emergency Vehicles", 978-1-53865257-2/18/\$31.00 (C2018 IEEE

[7] R Vani , N Thendral , J C Kavitha , N P G Bhavani," Intelligent Traffic Control System with Priority to Emergency Vehicles", IOP Conf. Series: Materials Science and Engineering 455 (2018) 01202

[8] Rahul Pundir, Vikash Kumar Yadav, Sunil Prakash Mandrawal, Deepak Kumar, "Smart Traffic System for Emergency Vehicle", International Journal of Science, Engineering and Technology, 2017, Volume 5 Issue 6 ISSN (Online): 2348-4098.

[9] Khalil M. Yousef, Jamal N. Al-Karaki, "Intelligent Traffic Light Flow Control System Using Wireless Sensors Networks", Journal Of Information Science And Engineering 26, 753-768 (2010). 